\title{
Mapping the depth to magnetic basement using inversion of pseudogravity: Application to the Bishop model and the Stord Basin, northern North Sea
}

\author{
Ahmed Salem ${ }^{1}$, Chris Green ${ }^{2}$, Samuel Cheyney ${ }^{3}$, J. Derek Fairhead ${ }^{2}$, \\ Essam Aboud ${ }^{4}$, and Simon Campbell ${ }^{3}$
}

\begin{abstract}
Magnetic depth estimation methods are routinely used to map the depth of sedimentary basins by assuming that the sediments are nonmagnetic and underlain by magnetic basement rocks. Most of these methods generate basement depth estimates at discrete points. Converting these depth estimates into a grid or map form often requires the application of qualitative methods. The reason for this is twofold: first, in deeper parts of basins, there is generally a scarcity of depth estimates and those that have been determined tend to be biased toward the shallower basement structures close to the basin edge; and second, depth estimates intrinsically relate to magnetic anomalies that emanate from the top edges of basement faults/contacts resulting in a shallow depth bias. Thus, simple grid interpolation of these depth estimates often forms a shallower and structurally unrepresentative map when evaluated in detail. To overcome these problems of qualitative and/or simple grid interpolation of these point-depth estimates into a regular grid, we use the pseudogravity field transform response of the magnetic field to constrain this interpolation using inversion methods together with the relationship between the point-depth estimates and their pseudogravity values. The pseudogravity transformation converts a grid of magnetic data such that the resulting grid has the same simple relationship to magnetic susceptibility that a gravity grid has to density. The pseudogravity map is thus straightforward to visualize in terms of basement structure, but it only maps the magnetic properties of the subsurface and is not related to the gravity anomaly or the density. We describe a practical approach to invert pseudogravity grids using gravity inversion software to produce a 3D basin model assuming a constant susceptibility basement. The approach is initially tested on the Bishop 3D model and then applied to an example from the northern North Sea. This approach can be considered complementary to 3D gravity inversion and has the advantage that the pseudogravity response is not affected by structure within the sediments or effects such as sediment compaction, inversion, or isostatic compensation, all of which often complicate the gravity response of sedimentary basins.
\end{abstract}

\section{Introduction}

One of the prime uses of the magnetic method in oil exploration is as a cost-effective tool to map the basement surface beneath sediments. Depth to basement mapping is an important control in the analysis of frontier sedimentary basin areas. A range of semiautomated depth estimation methods including Euler deconvolution (Reid et al., 1990), source parameter imaging (Thurston and Smith, 1997), and tilt-depth (Salem et al., 2007) are now routinely used to facilitate this process. These tools do not make direct assumptions about magnetic properties, but must generally be tuned to particular shapes of geologic features (e.g., via a "structural index"). All of these methods tend to identify the top edges/corners of magnetized geologic structures that have vertical extent (dikes, faults, and contacts) due to the fact that they rely on first- or second-order derivatives of the magnetic field, which enhance the shorter wavelength anomalies and hence the shallower features. Thus, for sedimentary basins, the basement flanks are generally well-resolved whereas deeper structures within the basin typically are not. The implication of this is that the actual depths of the deepest parts of sedimentary basins are often missed

\footnotetext{
${ }^{1}$ Getech, Leeds, UK; University of Leeds, School of Earth and Environment, Leeds, UK; and Nuclear Materials Authority, Cairo, Egypt. E-mail: ahmedsalem30@yahoo.com.

${ }^{2}$ Getech, Leeds, UK and University of Leeds, School of Earth and Environment, Leeds, UK. E-mail: cmg@getech.com; derek.fairhead@ getech.com.

${ }^{3}$ Getech, Leeds, UK. E-mail: Samuel.Cheyney@getech.com; Simon.Campbell.Getech.com.

${ }^{4}$ King Abdulaziz University, Faculty of Earth Sciences, Jeddah, Saudi Arabia. E-mail: essamaboud@yahoo.com.

Manuscript received by the Editor 10 July 2013; revised manuscript received 11 October 2013; published online 21 March 2014; corrected version published online 3 April 2014. This paper appears in Interpretation, Vol. 2, No. 2 (May 2014); p. T69-T78, 14 FIGS.

http://dx.doi.org/10.1190/INT-2013-0105.1. @ 2014 Society of Exploration Geophysicists and American Association of Petroleum Geologists. All rights reserved.
} 
or underestimated by such depth-estimation methods and are not well-represented in any interpolated grid version of these depth estimates.

In contrast to the calculation of the derivatives of the magnetic field, the pseudogravity transform (Baranov, 1957 ) is an integral transformation which enhances the longer wavelengths of the magnetic field and images the bulk shape of magnetic bodies. The transform essentially consists of a reduction to pole operation to center the (assumed) induced magnetic anomalies over their causative bodies and a vertical integration to simplify the anomaly such that the pseudogravity anomaly due to a uniformly, induced magnetized body has the same shape as the gravity anomaly due to the same body with uniform density. The pseudogravity is thus essentially the same as the magnetic potential for a vertical magnetic field. Pseudogravity has been used to map contacts (Fairhead et al., 2004; Pilkington, 2007), as an alternative domain for comparing observed and modeled magnetic fields (Kimbell et al., 2010) or for direct comparison of gravity and magnetic anomalies (Reeh and Aifa, 2008). The pseudogravity can be analyzed and modeled in the same manner as gravity data, but it remains a magnetic anomaly and can only be interpreted in terms of magnetization distribution. In practice, pseudogravity is related to magnetic susceptibility as induced magnetization is inherently assumed in the reduction to pole process. There is, however, no need to assume any relationship between density and susceptibility to interpret the pseudogravity.

In this study, we devise and test a practical approach to $3 \mathrm{D}$ inversion of the pseudogravity to map basement relief. We apply algorithms and software that are commonly used in gravity inversion to interpret pseudogravity data in terms of depth-to-basement. We define an appropriate magnetization for the inversion based on direct depth information from a finite number of control points. The final 3D model now uses the complete information from the magnetic field as well as control data and selected magnetic depth estimates to constrain the depth model.

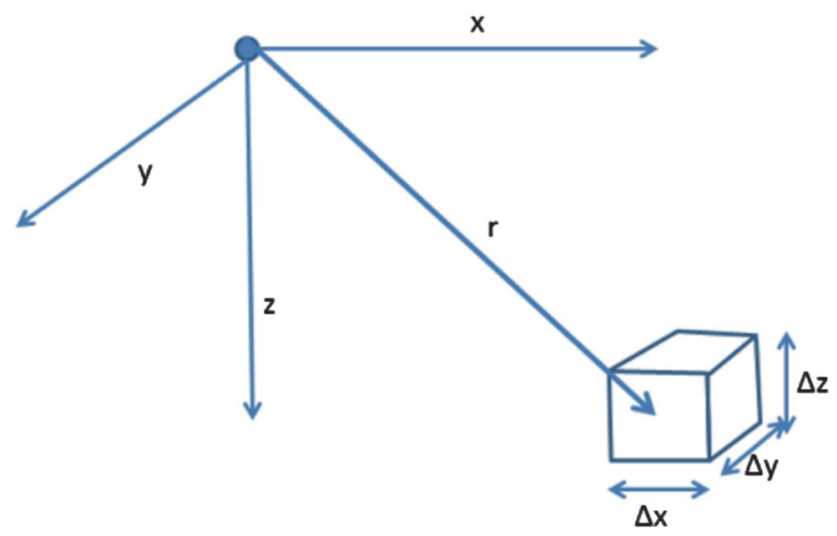

Figure 1. Basic element for calculating magnetic or gravity fields.

\section{Relationship between pseudogravity and gravity}

To apply gravity tools (especially 3D inversion) to pseudogravity data, it is necessary to scale the data appropriately. Applying the correct scale factor to the vertical integral (VI) of the reduced-to-pole gravity field allows the "density" values in the pseudogravity modeling/inversion to be directly interpreted as susceptibility.

Here, we consider the gravity and magnetic effect of a small or infinitessimal volume size $\Delta x, \Delta y$, and $\Delta z$ at location $x, y$, and $z$ relative to the observation point (Figure 1), where $z$ is the vertical direction and $x$ and $y$ are horizontal coordinates. Talwani and Heirtzler (1964) and also Talwani (1965) use this as the element which is integrated to calculate the magnetic field of more complex shapes. Talwani and Heirtzler (1964) are the basis for almost all 2D magnetic modeling programs. Using our own nomenclature, Talwani and Heirtzler (1964) and Talwani (1965) have

$$
\begin{gathered}
U=\frac{\mathbf{m} \cdot \mathbf{r}}{r^{3}}, \\
\mathbf{m}=\mathbf{J} . \Delta x \cdot \Delta y . \Delta z, \\
\mathbf{J}=k \cdot \mathbf{F},
\end{gathered}
$$

where $U$ is magnetic potential (i.e., the same as the pseudogravity for vertical field and magnetization), $\mathbf{m}$ is magnetic moment, $\mathbf{r}$ is vector from the observation point to the source, $r$ is scalar distance, $\mathbf{J}$ is magnetization $\left(J_{x}, J_{y}, J_{z}\right), k$ is susceptibility (in cgs magnetic units), and $\mathbf{F}$ is ambient magnetic field (in nT). Thus,

$$
U=\frac{J_{x} \cdot x+J_{y} \cdot y+J_{z} \cdot z}{r^{3}} \cdot \Delta x \cdot \Delta y \cdot \Delta z .
$$

For vertical field and magnetization, the VI of the RTP is given by

$$
V I=U=\frac{J_{z} \cdot z}{r^{3}} \cdot \Delta x . \Delta y . \Delta z=\frac{k \cdot F \cdot z}{r^{3}} \cdot \Delta x . \Delta y . \Delta z .
$$

The gravity field $g$ for the same element is given by

$$
\begin{gathered}
g=\frac{G \cdot M \cdot z}{r^{3}} \\
M=\rho . \Delta x \cdot \Delta y \cdot \Delta z,
\end{gathered}
$$

where $M$ is the mass, $G$ is the gravitational constant $\left(\sim 6.67384 \times 10^{-11} \mathrm{~m}^{3} \cdot \mathrm{kg}^{-1} \cdot \mathrm{s}^{-2}\right)$, and $\rho$ is the density. Hence,

$$
g=\frac{G \cdot \rho . z}{r^{3}} \cdot \Delta x \cdot \Delta y \cdot \Delta z .
$$

The ratio between VI and gravity is given by 


$$
\frac{V I}{g}=\frac{k}{\rho} \cdot \frac{F}{G} .
$$

So, if $F=50,000 \mathrm{nT}=5 \times 10^{-5} \mathrm{~T}$ and for VI in T.m, $g$ in $\mathrm{m}_{\mathrm{s}} \mathrm{s}^{-2}, \rho$ in $\mathrm{kg} . \mathrm{m}^{-3}$

$$
\frac{V I}{g} \approx 749193.9 \frac{k}{\rho} .
$$

For $V I$ in nT.m, $g$ in mGal, and $\rho$ in $g . \mathrm{cm}^{-3}$

$$
\begin{gathered}
\frac{V I}{g} \approx 7491939 \frac{k}{\rho} \\
\frac{V I}{g} \approx 149.8 F \frac{k}{\rho} .
\end{gathered}
$$

Equations 5 and 8 are clearly the same, geometrically, which implies that integrating these expressions over a $3 \mathrm{D}$ volume will yield proportional VI and $g$ for any shape body and hence the pseudogravity transformation is valid for any geologic situation. The scale factor for the basic element is thus appropriate for all body shapes. Therefore, a VI (of the RTP) in $\mathrm{nT} \cdot \mathrm{m}$, should be divided by $(149.8 F)$ where $F$ is the ambient field in nT to generate usable pseudogravity. It is then possible to model or invert the pseudogravity as though it was gravity in mGal, but based on cgs susceptibility units rather than density in $g . \mathrm{cm}^{-3}$,

$$
P S G=\frac{V I}{149.8 F} .
$$

By analogy with the Bouguer slab formula, the pseudogravity effect of an infinite slab of thickness $Z(m)$ and susceptibility $k$ can thus be expressed as

$$
P S G_{\text {slab }}=2 \pi G . k . Z \approx 0.04193 k . Z .
$$

Using the $1 \mathrm{D}$ assumption, the susceptibility contrast $\Delta k$ can be estimated from the slope of a graph of pseudogravity against depth. For pseudogravity difference $\triangle P S G$ and depth difference $\Delta Z$,

$$
\Delta k \approx \frac{\Delta P S G}{0.04193 . \Delta Z} \approx 0.1592 \frac{\Delta V I}{F . \Delta Z} .
$$

\section{Test on theoretical data}

To test our 3D pseudogravity inversion approach, we use the Bishop 3D basement model. The Bishop model has been used by various authors to test methods of estimating source depths from magnetic data (Williams et al., 2002, 2005; Fairhead et al., 2004; Reid et al., 2005); it was the subject of a workshop at the 2006 SEG Annual International Meeting. The aim of the Bishop model was to provide a data set that shares the complexity of real basement relief, while allowing depth estimate results to be directly compared to the model depths. The model is derived from a digital elevation model (DEM) for a $10.5 \times 10.5 \mathrm{~km}$ area of the Volcanic Tablelands close to Bishop, California, that has been scaled up to produce a 3D test model with dimensions $315 \times 315 \mathrm{~km}$ on a $500-\mathrm{m}$ grid representative of a basinscale magnetic basement surface. The topographic surface was then shifted down such that the highest point has a depth of a few hundred meters below datum and the deepest point lies just above 10-km depth. In this model, all parts shallower than the basement surface are considered nonmagnetic and those below the basement surface are assigned a constant susceptibility of 0.001 cgs units. Figure 2a shows the basement topography of the Bishop model. The corresponding calculated RTP magnetic anomaly is displayed in Figure 2b. The pseudogravity of the calculated magnetic response of the model is shown in Figure 2c.

The magnetic field over a sedimentary basin is largely derived from the underlying magnetic basement and is a complicated nonlinear function of the basement depth and the magnetization distribution within the basement. Even for the simple case where the magnetization of the basement can be considered constant, the relationship between basement depth and magnetic anomaly is nonlinear (Figure 3a) where, for example, a simple basement high can generate positive and negative anomalies.

Although still nonlinear, the pseudogravity has the same simple relationship with magnetic bodies that gravity anomalies have with bodies of anomalous density (Figure 3b). If we assume that the magnetic anomaly is produced by basement topography, the pseudogravity at any point on the grid will be proportional to the susceptibility and for relatively low basement relief will be approximately proportional to the depth of the basement. Figure $3 \mathrm{~b}$ shows that this is a reasonable approximation in practice. This simple relationship is an attraction of working with pseudogravity because it is much easier to visualize the relationship between anomaly and depth and hence identify problems in the process. Also, given a sufficient number of points at a range of depths, the susceptibility (proportional to the slope) could be calculated using equation 15.

The offset on the pseudogravity axis is related to the constant of integration in calculating the pseudogravity. This suggests that a simplified 1D approximation that is analogous to the 1D Bouguer correction applied in gravity data reduction could be used to estimate the pseudogravity at each basement control point (using equation 14) and hence constrain the inversion. This will make it possible to use 3D gravity inversion software to map the basement depth, given appropriate values for susceptibility. Space domain (e.g., Cordell and Henderson, 1968) and Fourier domain (e.g., Oldenburg, 1974) algorithms are commonly used for 3D gravity inversion; in this study, we use a Fourier approach implemented in GMSYS-3D ${ }^{\mathrm{TM}}$ software. This software implements the Fourier domain calculation of 
Parker (1973) to invert for a single constant density contrast surface.

To invert the pseudogravity data (Figure 2c), we need a realistic estimate of the susceptibility to recover the correct depth to basement. Susceptibility values can be estimated based on the slab formula with some

a)
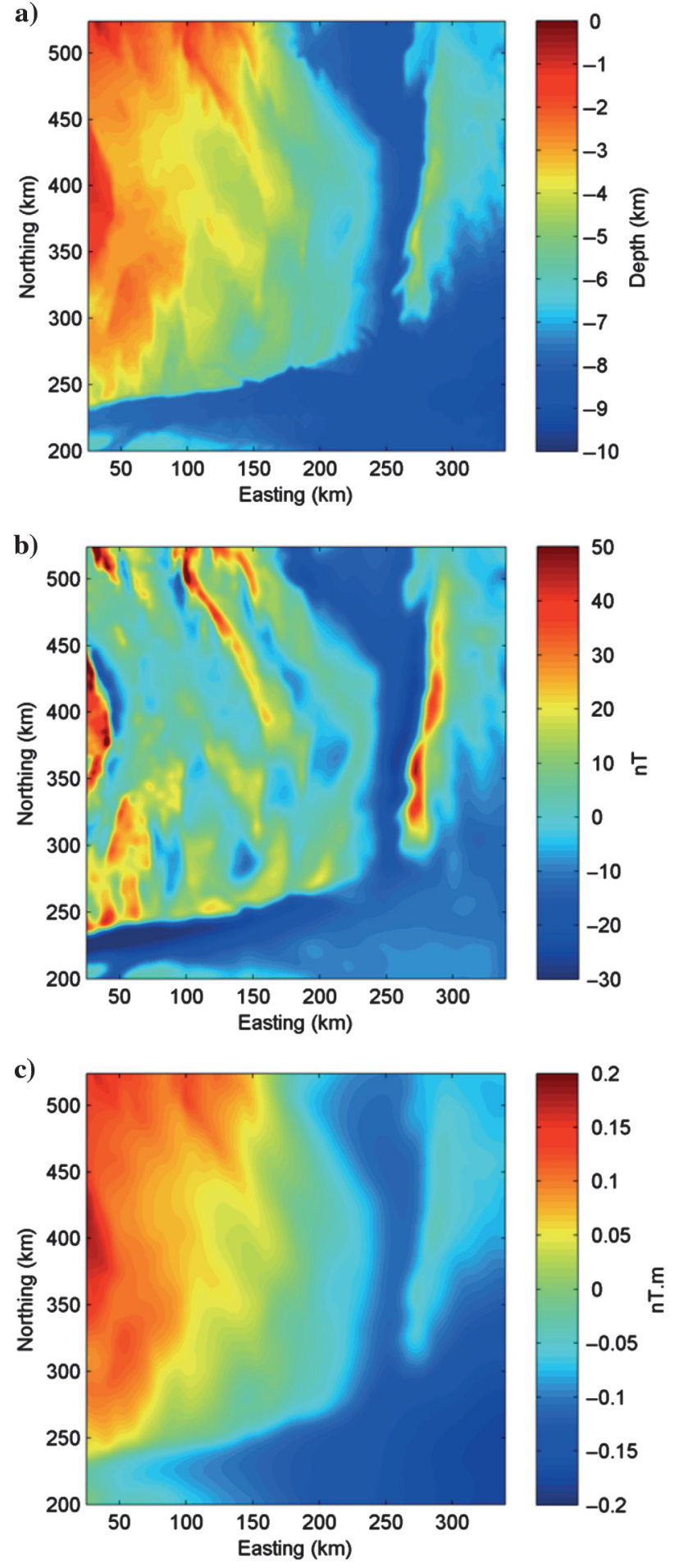

Figure 2. (a) Bishop model depth. (b) Corresponding calculated RTP magnetic anomaly. (c) Pseudogravity of the Bishop model magnetic anomaly. depth controls such as basement outcrop, wells, and seismic depths. When such depth controls are not available, we can use the best depth estimates calculated from the magnetic data themselves. This approach to using control points effectively assumes that the 1D pseudogravity from the slab formula is equivalent to the gravity anomaly at the same point due to the full $3 \mathrm{D}$ basement surface; this assumption is reasonably accurate in areas of flat-lying geology, but local peaks and troughs do not make good control points and control points which are representative of the area as a whole should be used where possible. Comparison of control depths against inverted depths at the same points helps to identify whether this process has worked satisfactorily. In this example, we constrain the depth and the susceptibility based on 10 arbitrary (but well-distributed) basement depth values extracted directly from the depth grid (Figure 4a); these could be considered to represent a set of basement wells within the study area. The crossplot (Figure $4 \mathrm{~b}$ ) shows a strong linearity (R-squared value of 0.98 ) in the relationship

a)
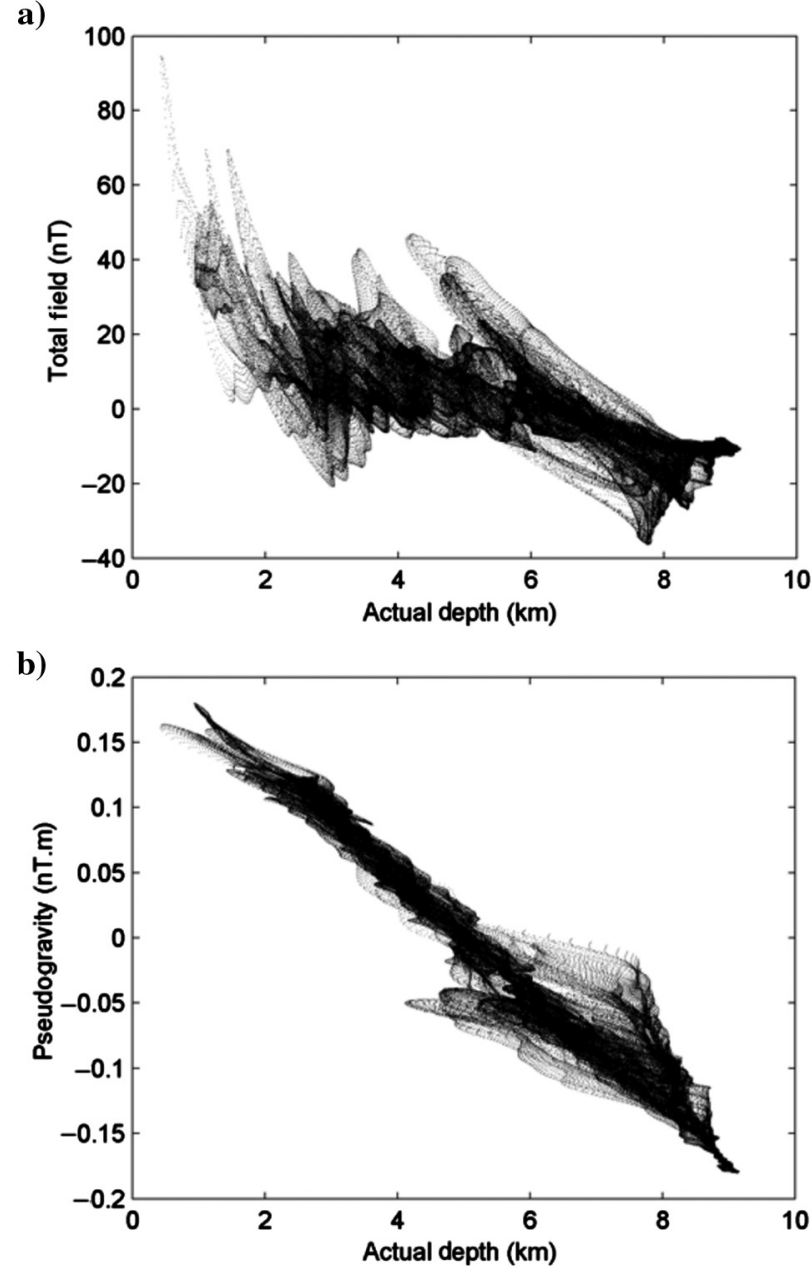

Figure 3. (a) One-dimensional crossplot of RTP magnetic data against depth for the Bishop model, showing the nonlinear relationship. (b) Pseudogravity against depth, showing a strong linearity in the relationship between the two. 
between pseudogravity and control point depth. The best-fit line in the crossplot can be characterized by a slope and an intercept. The intercept $(0.21 \mathrm{nT} \cdot \mathrm{m})$ is effectively the constant of integration in the pseudogravity calculation and is the value that should be subtracted from the pseudogravity grid prior to the $3 \mathrm{D}$ inversion; in a real case, this regional trend may take the form of a slope or a more complex surface. The slope is proportional to the susceptibility of the model; in this case, the slope implies (based on equation 15) a susceptibility of $0.001 \mathrm{cgs}$ units, matching the value which was used in the generation of the model. The pseudogravity data were then inverted for a basement surface with a constant susceptibility contrast of $0.001 \mathrm{cgs}$. Figure $5 \mathrm{a}$ shows the result of inverting the pseudogravity data as a depth map. Comparison of the depth estimates and real depths is displayed in Figure 5b. It is clear that there is a very good correlation between real and inverted depth increasing well into the range of the greatest depths. The inversion depth map also retains much of the character observed in the Bishop model.

a)

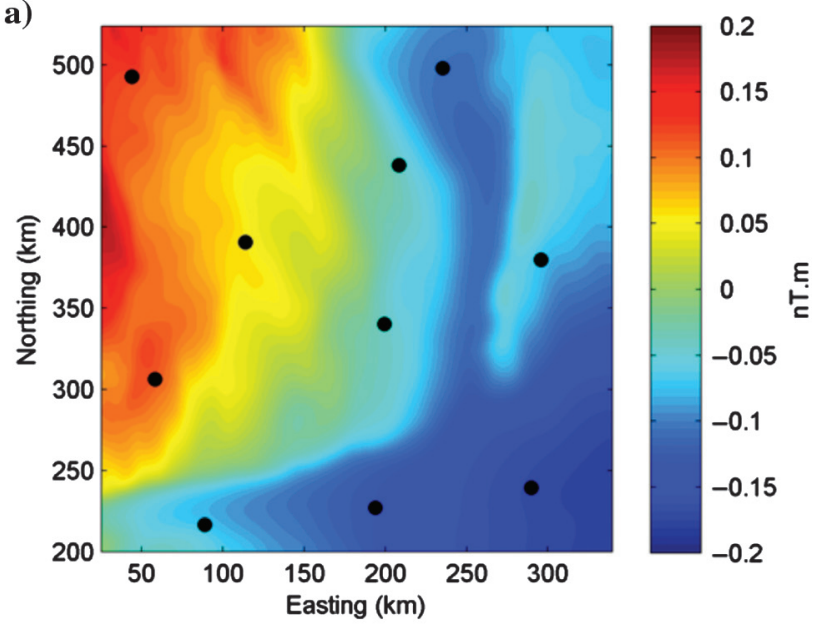

b)

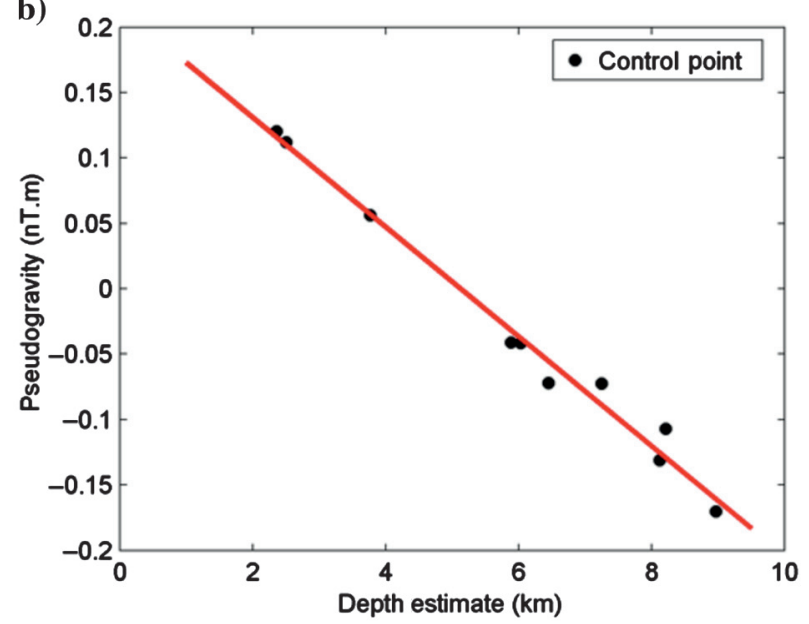

Figure 4. (a) Location and (b) depth estimates from 13 selected anomalies, using the control points. Note the strong linearity between the pseudogravity and the selected depths.
An alternative approach based on derivatives of the magnetic field would be to use the SPI method to generate a depth grid for the whole area. Figure 6a shows the result of making SPI depth estimates based on every local maximum of the local wavenumber; 1605 points in all have been interpolated using minimum curvature to generate this grid, using a 5000-m cell size. Minimum curvature is not generally considered ideal for producing depth to basement grids, but in this case, the number and distribution of depth estimates means that the gridding algorithm has little impact. The depth map resolves less detail and is generally shallower than the corresponding result from pseudogravity inversion (Figure 5a). Direct comparison of the SPI depths with model depths (Figure 6b) shows (as expected) a generally good match at shallow depths (up to $\sim 4 \mathrm{~km}$ ), but poor imaging of basement depth in the deeper areas; the shallowest depths are not well imaged by
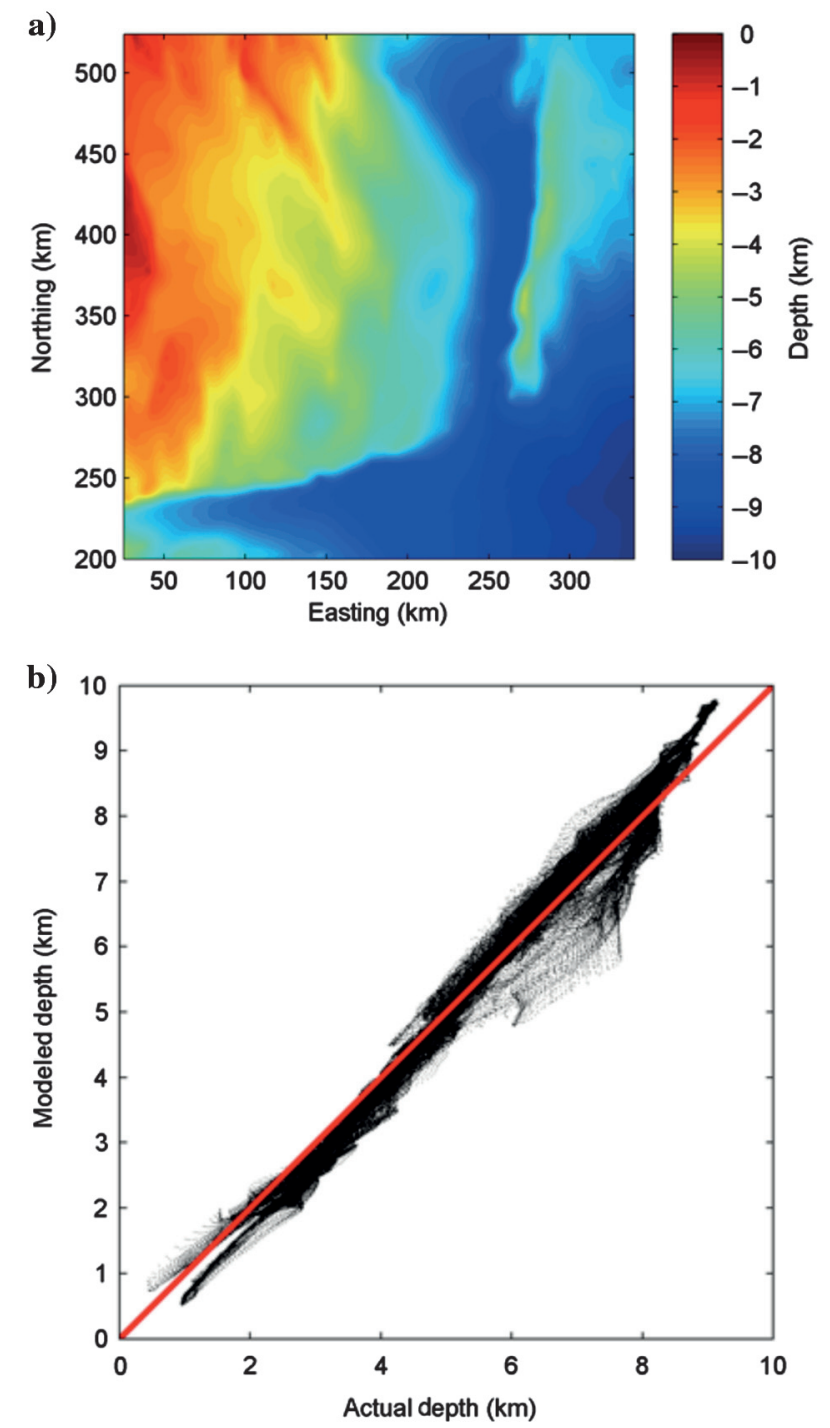

Figure 5. (a) Depth to basement for the Bishop model, using 3D inversion of the pseudogravity data. (b) Crossplot of the modeled depth-to-basement against the true depth to basement. 
SPI — probably due to limited high-frequency content imposed by the 500-m cell size of the data. The differences between the actual Bishop Model and the surfaces generated from the pseudogravity inversion and the gridded SPI solutions have been calculated (Figure $7 \mathrm{a}$ and $7 \mathrm{~b}$, respectively). The pseudogravity inversion grid is, overall, much more representative of the whole basement surface than the SPI-derived grid. This indicates that the pseudogravity inversion method can be useful in addressing one of the limitations of the more conventional derivative-based depth to source methods which is the bias toward a large number of shallow solutions that are also more accurate than deeper solutions.

\section{Test on real data}

We demonstrate the practical application of the approach using a magnetic data set over the southern edge of the Stord Basin, northern North Sea (Figure 8a). This area has previously been interpreted by Hospers
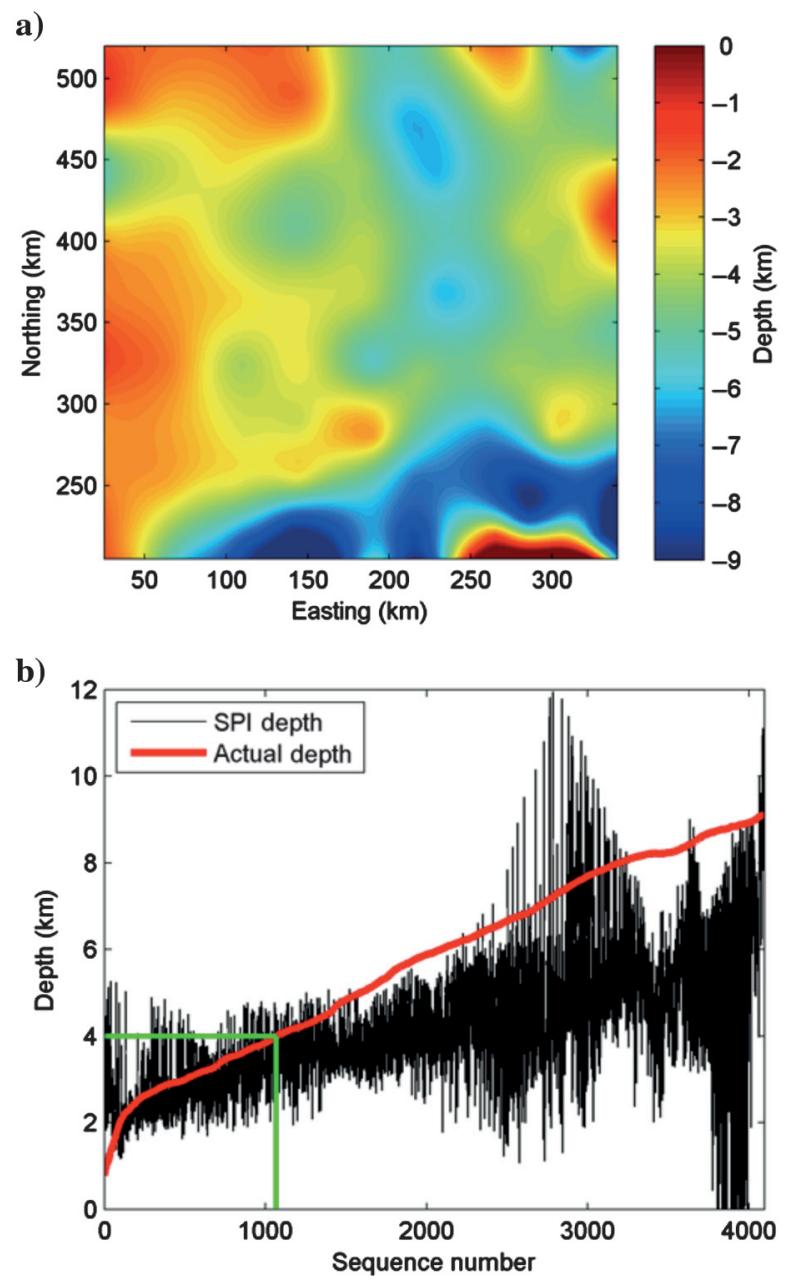

Figure 6. (a) Depth to basement for the Bishop model using the automated SPI approach based on 1605 depth estimates. (b) Depths from the SPI grid and actual model depths; note that the SPI depths are close to the actual depths up to 4-km depth, but they significantly underestimate the depth in deeper areas. and Ediriweera (1991) using seismic data (Figure 8b). The dominant structural feature in this area is a faultbounded north-south trending basin (the Stord Basin) with a broad high to the east and a more local high (the Utsira High) to the west (Hospers and Ediriweera, 1991).

The magnetic data (Figure 9) were sampled at a grid interval of $1 \mathrm{~km}$ from a recent compilation of magnetic data for Europe (Fletcher et al., 2011). The grid is continued to $1 \mathrm{~km}$ above sea surface and in this area is based on all available magnetic data to generate a unified $1 \mathrm{~km}$ grid of total magnetic intensity (TMI). Figure 10 shows all the SPI (Thurston and Smith, 1997) depth solutions derived from the magnetic data set. It can be seen that SPI depth estimates in the shallow basement area to the east generally match well with the mapped basement depths, whereas those in the west and center of the area are much more variable; overall, the SPI depths do not give a good representation of the shape of the basin.
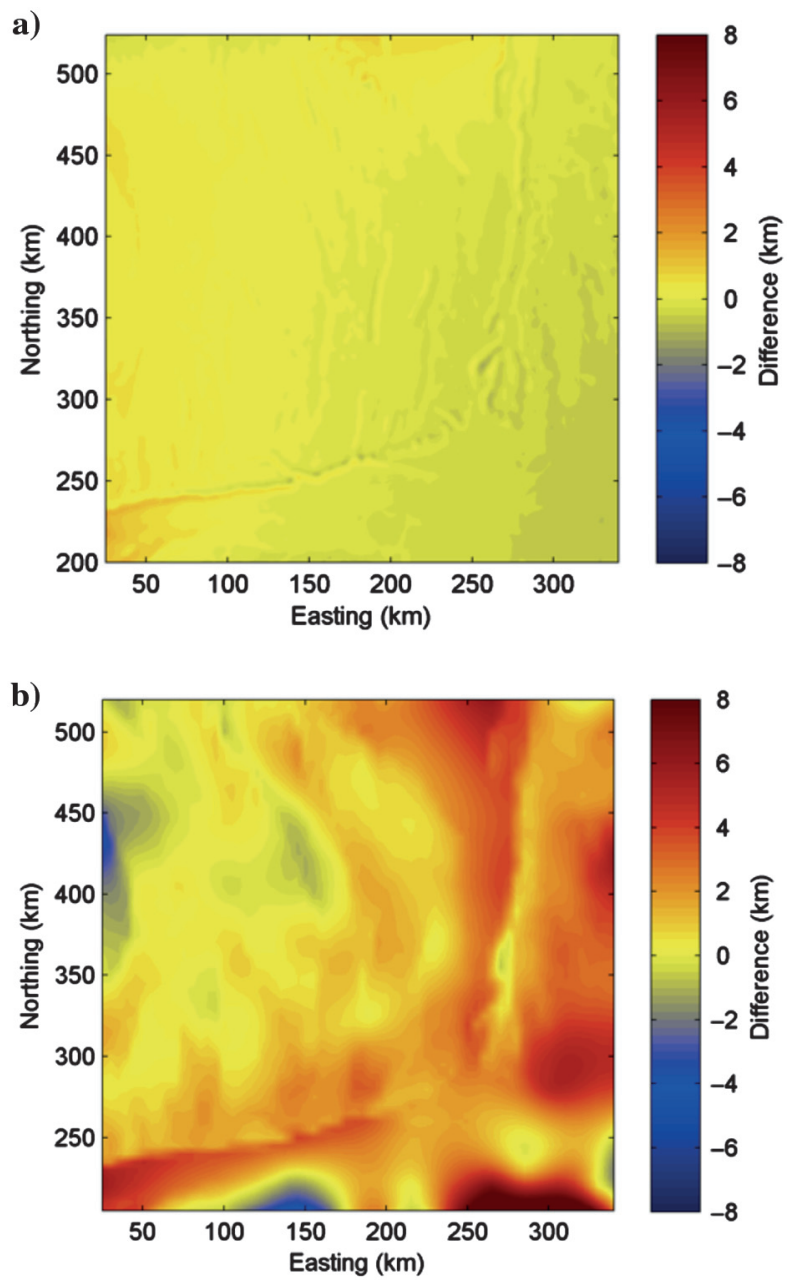

Figure 7. (a) Difference in depth between the pseudogravity inversion result and the actual Bishop model surface. (b) Difference in depth between the gridded SPI solutions and the actual Bishop model surface. 
To apply the present approach, the magnetic data of the Stord Basin study area are transformed into pseudogravity by vertical integration of the RTP field with scaling such that the resulting field can be interpreted in terms of susceptibility variation (equation 13). The pseudogravity map of the Stord Basin study area (Figure 11) enhances magnetic anomalies from deeper magnetic sources (longer wavelengths) and reduces the dominance of shallow magnetic sources (shorter wavelengths).

In this example, we assume that the sediments are nonmagnetic and invert for a constant susceptibility basement; based on scaling in the pseudogravity transform, this susceptibility can be represented by the appropriate density in the gravity inversion software. As with a gravity inversion for a basin, there is a need to ensure that the pseudogravity grid to be inverted is wholly $\leq$ zero and that any control points are honored. If there are sufficient basement control points (e.g., wells) with a large enough depth range, then the susceptibility could be derived directly from the control data. In this study area, there is no outcropping basement and only two basement wells to provide direct control; we choose not to use the available seismicderived basement depths as control, but rather use them to assess the result from pseudogravity inversion. Otherwise, if no measured basement susceptibility data are available, the susceptibility value must be based on knowledge of the regional geology, or estimates from the magnetic data. Here, we use the SPI method (Thurston and Smith, 1997) to generate extra control points (Figure 11). We select the best-controlled SPI solutions based on the clarity and simplicity of the magnetic anomalies and the general consistency of the depth solutions with the pseudogravity map. Figure 11 shows the locations of the final control points (wells marked with closed symbols and SPI depths as open circles) with the depth to basement values. Figure 12 shows the scattergram between these control points and the pseudogravity data; there is a correlation between the two, but also considerable scatter $(R$-squared $=0.48)$. In this case, a susceptibility of $0.0019 \mathrm{cgs}$ units is derived directly from the slope of the best-fit straight line through the data points; no local susceptibility data are available to test this, but the value is in the general range for basement rocks.

We then use a simple slab formula (analogous to the Bouguer slab formula) to compute the expected pseudogravity at the control points. Next, we use a smooth surface based on the difference between the observed and computed values to adjust the pseudogravity data. In this process, where the regional field is not a flat plane, a susceptibility which allows a relatively simple regional adjustment is considered most suitable. The adjusted pseudogravity is shown in Figure 13; this is the pseudogravity grid actually used in the inversion.

Figure 14 shows the results of the 3D inversion from the pseudogravity data. The root-mean-squared errors between the inverted depths and the depths used at the control points is $0.05 \mathrm{~km}$. The results generally indicate a good correlation between the inversion depths and the depth to basement map of Hospers and Ediriweera (1991). The shape of the Stord Basin and the Utsira High match well and the depths are in generally good agreement. The edge of the high to the east is wellimaged, but there are some differences in depth across the eastern platform. It should be noted, however, that
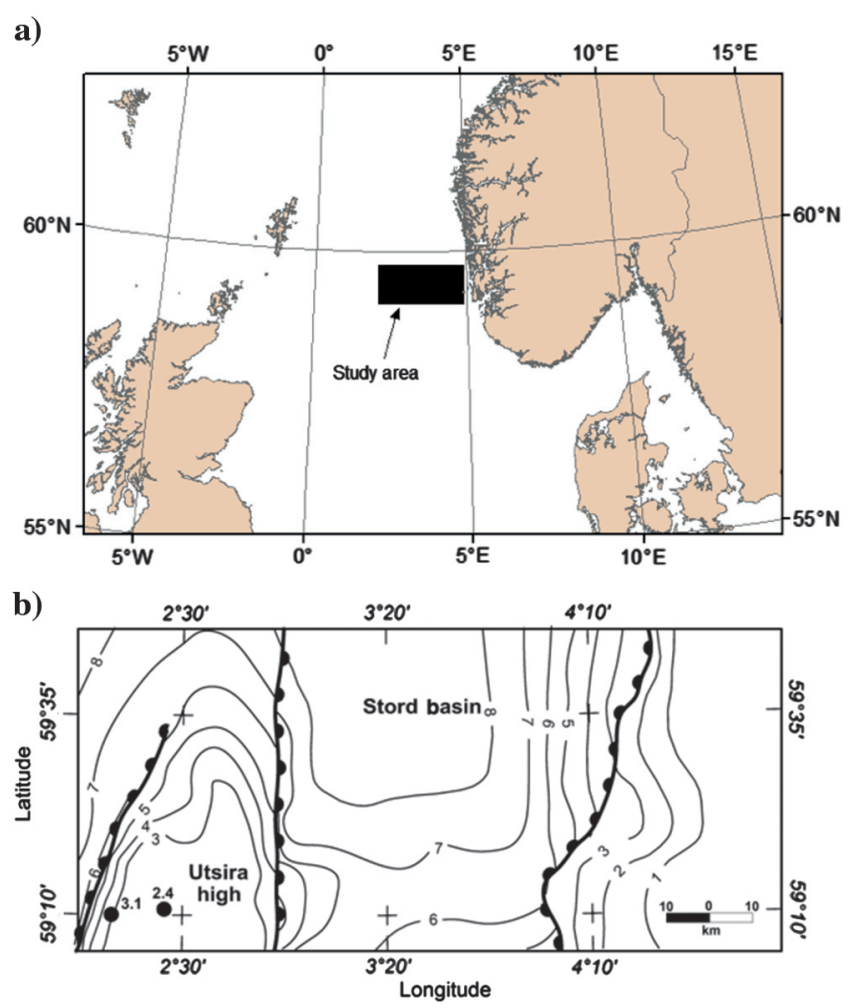

Figure 8. (a) Location of the study area at the southern edge of the Stord Basin in the northern North Sea. (b) Structural interpretation of the Stord Basin study area based on Hospers and Ediriweera (1991).
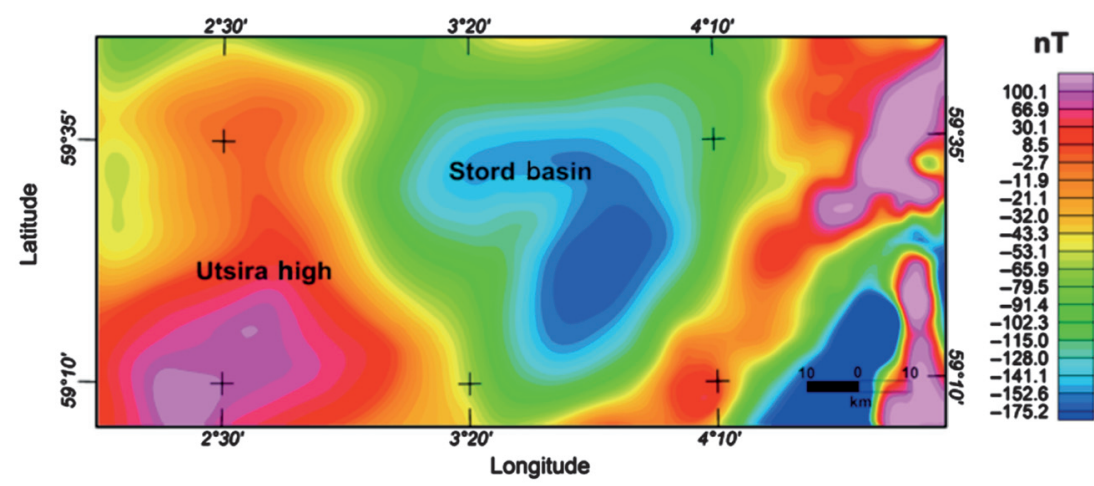

Figure 9. TMI anomaly map of the Stord basin. 


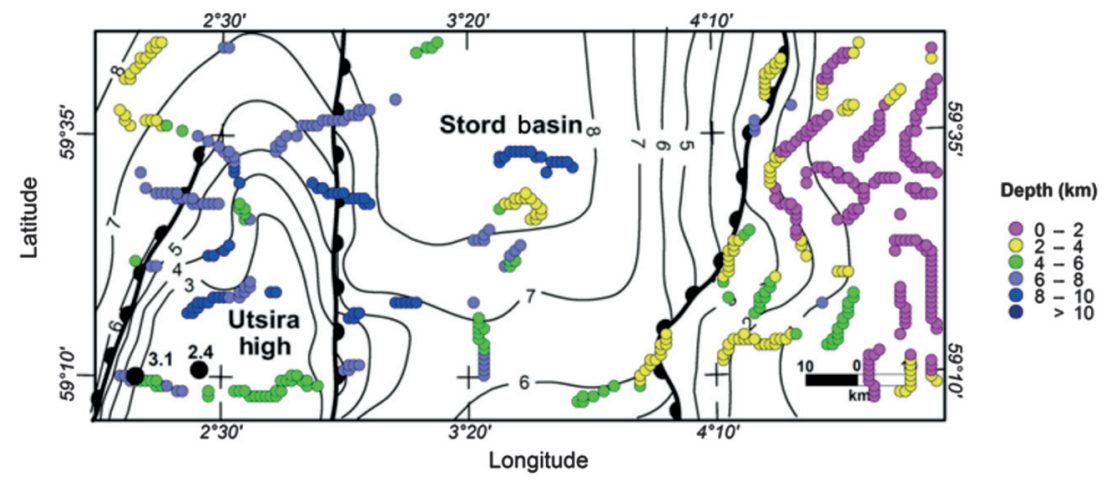

Figure 10. SPI solutions for the Stord Basin study area. Circles show the location of SPI solutions (colored according to depth).

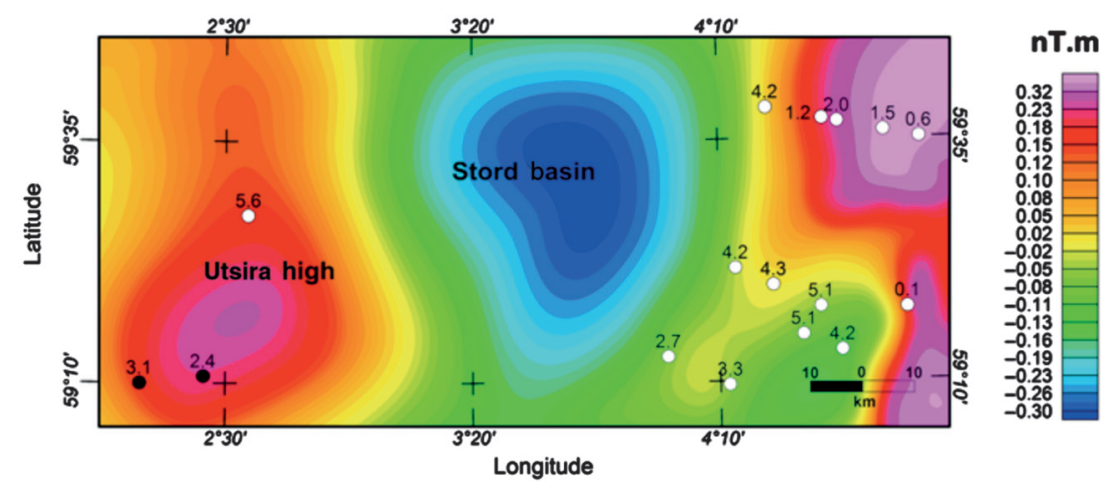

Figure 11. Pseudogravity of the Stord Basin study area showing the control points for estimating the susceptibility contrast to be used in the pseudogravity inversion. Solid circles show the location of well data and the associated depth to basement; open circles are SPI depths from carefully selected anomalies.
Hospers and Ediriweera (1991) have no data at this easternmost edge of their study area, so the local basement low around $4^{\circ} 25^{\prime} \mathrm{E}$ and $59^{\circ} 15^{\prime} \mathrm{N}$ may well not contradict any seismic data. Certainly, the depth grid from the pseudogravity inversion provides a much more representative basement depth map than could readily be derived from the SPI depth solutions shown in Figure 10 .

\section{Discussion}

The pseudogravity 3D inversion method described above is seen to work very effectively when applied to the Bishop model. In the Stord Basin area in the northern North Sea, the method also produces basement depths which generally fit well with previous depth to basement maps based on seismic data (these seismic data were not used to constrain the inversion in any way). Of course, the constant basement susceptibility of the Bishop model used here makes it an ideal case for this simple constant susceptibility inversion. The Stord Basin example may also be close to an ideal case; the constant susceptibility inversion fits well with known and expected basement depths. Pseudogravity inversion with laterally varying basement susceptibility could readily be implemented (e.g., in GMSYS-3D ${ }^{\mathrm{TM}}$ software), however, unless there are sufficient control data points across the area of susceptibility variation, it would not be possible for the susceptibility to be identified and constrained. This is analogous to 3D gravity inversion where varying density contrast can readily be incorporated in the inversion, but constraining the density variation is less straightforward.

The use of SPI depths as control points in the Stord Basin study area is not considered ideal. As has been shown (Figure 6b), SPI depths are often in error especially in deeper areas and if these control depths are wrong, the depths from the inversion will be wrong in those areas. On the other hand, if fewer depth control points are used and there are gaps in their coverage, then there may be considerable error in the long wavelength grid that is removed, which will also result in errors in the inverted depth grid. SPI depths from a small number of handpicked anomalies will generally have greater confidence than automatically generated SPI solutions, and hence these depth estimates are likely to provide useful control in areas where hard control data points are sparse.

Gravity maps are often seen to be significantly different from pseudogravity maps and are often more complex and therefore more complicated to invert. This is
Figure 12. Crossplot showing the relation between the control point depths and the pseudogravity data. 
likely to be due to varying density sediments within the basin. This is a clear potential advantage of pseudogravity over gravity, but there may be cases where the reverse is true - e.g., where sedimentary densities vary smoothly, while basement densities have rapid variations. It is not yet clear how frequently the constant susceptibility approach will give satisfactory results, but it may well commonly be valid - at least for relatively small basins or subbasins.

Reducing magnetic data to the pole and hence calculating pseudogravity assumes that the magnetization of rocks is in the same direction as the earth's magnetic field. If magnetization is purely induced, this condition should be met, but in practice any recent remanence (parallel or antiparallel to the earth's field) should not stop the technique from working. In this case, the calculated susceptibility should actually be considered as an "effective susceptibility," which includes the effects of remanent magnetization. Large remanence in a different direction will adversely affect pseudogravity inversion as well as some semi-automated magnetic depth estimators.

Magnetic derivative methods rely on accurate data at short wavelengths so that the derivatives (and their products, quotients, etc.) can be confidently calculated; however, they can be calculated from relatively small areas of data. Pseudogravity, on the other hand, does not require such accurate magnetic data at short wave-

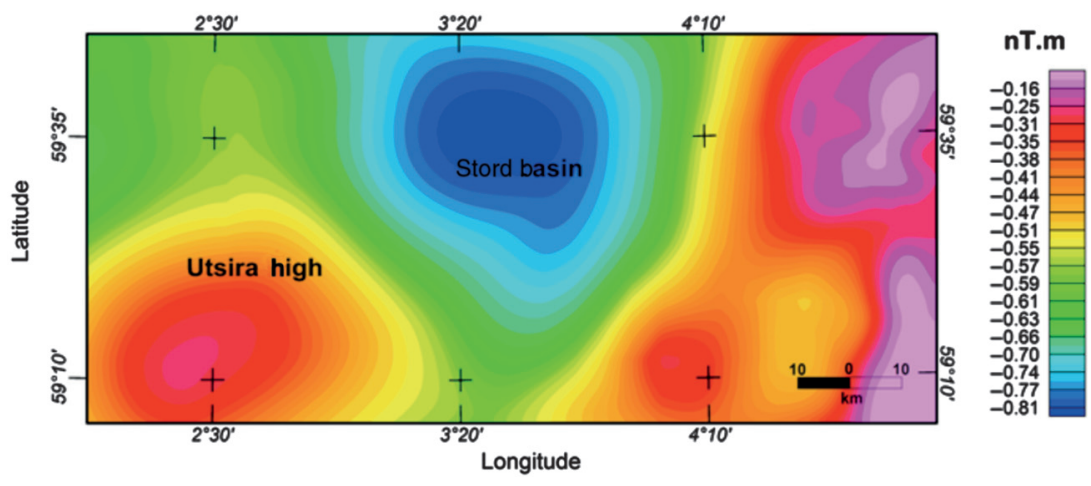

Figure 13. Adjusted pseudogravity; this is the grid that is actually used in the inversion.

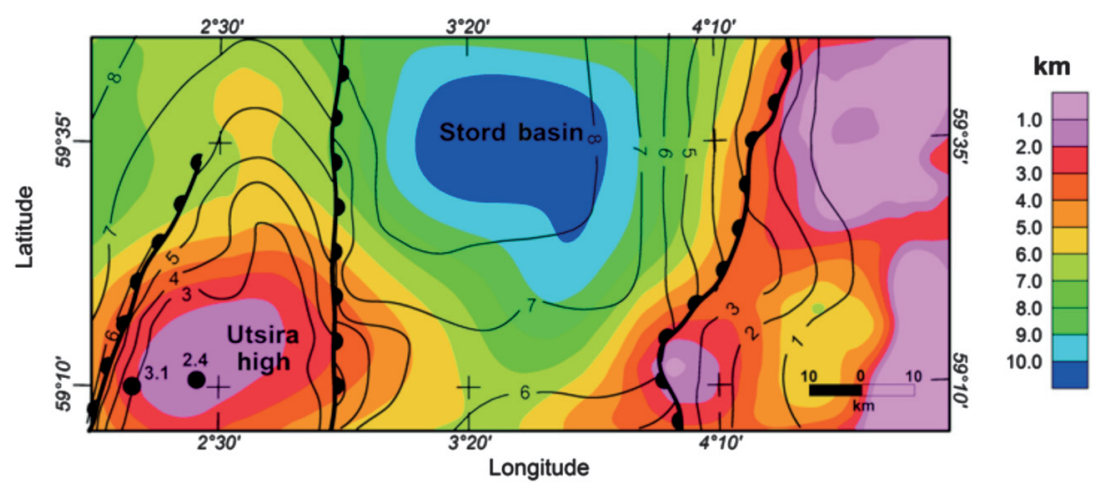

Figure 14. Depth to basement of the Stord Basin study area from the 3D inversion of pseudogravity. length, but because the long wavelengths are enhanced in the vertical integration, the data set should extend to some distance from the area of interest. Thus, there are a range of differences between 3D pseudogravity inversion and gravity inversion or magnetic derivative methods. As illustrated in this paper, the combination of magnetic depth estimators with pseudogravity inversion can be used to effectively map depth to basement, provided that the magnetic depth estimations are accurate and reliable. The addition of gravity inversion to the process may offer further improvements in accuracy and confidence in the results.

\section{Conclusion}

This simple approach to mapping depth-to-basement from 3D inversion of pseudogravity has been shown to be very effective. For a known model of realistic complexity, the depth and susceptibility are interpreted with good accuracy. Using real data from the northern North Sea, the correlation of the results to the published and recorded geologic information indicates that not only the approach, but also the application of the process and the susceptibility values used are reasonable and can provide significant insights into the basement morphology of the Stord Basin area. The combination of well depths and magnetic depth estimates at a small number of locations with pseudogravity inversion has been shown to be appropriate in this study with the pseudogravity inversion controlling the shape of the basement surface between robust depth estimates. It is clear that, as with gravity inversion, careful selection and application of available control data is essential to the success of the process. The variability of the magnetic properties of basement rocks might be expected to make this process problematic. Inversion of gravity data over sedimentary basins would be expected to be complicated by the effects of intrasedimentary structures, spatial and depth density variations within the basin, and isostatic processes. Thus, 3D pseudogravity inversion together with depth estimates could be used more generally, along with control data and possibly $3 \mathrm{D}$ gravity inversion, to increase the confidence of basement mapping from magnetic data and hence reduce exploration uncertainties.

\section{Acknowledgments}

We are grateful for comments by the associate editor, anonymous reviewer, Ran Zhang, and Alan Aitken that helped to improve the manuscript. We are also grateful for the help of David Blackledge in the production of the figures and illustrations. 


\section{References}

Baranov, V., 1957, A new method for interpretation of aeromagnetic maps: Pseudo-gravimetric anomalies: Geophysics, 22, 359-382, doi: 10.1190/1.1438369.

Cordell, L., and R. G. Henderson, 1968, Iterative threedimensional solution of gravity anomaly data using digital computer: Geophysics, 33, 596-601, doi: 10.1190/1 .1439955 .

Fairhead, J. D., S. E. Williams, and G. Flanagan, 2004, Testing magnetic local wavenumber depth estimation methods using a complex 3D test model: 74th Annual International Meeting, SEG, Expanded Abstracts, 742745 .

Fletcher, K. M. U., J. D. Fairhead, A. Salem, K. Lei, C. Ayala, and P. L. M. Cabanillas, 2011, Building a higher resolution magnetic database for Europe for resource evaluation: First Break, 29, 41-47.

Hospers, J., and K. K. Ediriweera, 1991, Depth and configuration of crystalline basement in the Viking Graben area, North Sea: Journal of the Geological Society, 148, 261-265, doi: 10.1144/gsjgs.148.2.0261.

Kimbell, G. S., J. D. Ritchie, and A. F. Henderson, 2010, Three dimensional gravity and magnetic modelling of the Irish sector of the NE Atlantic margin: Tectonophysics, 486, 36-54, doi: 10.1016/j.tecto.2010.02.007.

Oldenburg, D. W., 1974, The inversion and interpretation of gravity anomalies: Geophysics, 39, 526-536, doi: 10 $.1190 / 1.1440444$.

Parker, R. L., 1973, The rapid calculation of potential anomalies: Geophysical Journal of the Royal Astronomical Society, 31, 447-455, doi: 10.1111/j.1365-246X .1973.tb06513.x.

Pilkington, M., 2007, Locating geologic contacts with magnitude transforms of magnetic data: Journal of Applied Geophysics, 63, 80-89, doi: 10.1016/j.jappgeo.2007.06.001.

Reeh, G., and T. Aifa, 2008, Age of the source of the Jarrafa gravity and magnetic anomalies offshore Libya and its geodynamic implications: Journal of Geodynamics, 45, 217-233, doi: 10.1016/j.jog.2008.01.001.
Reid, A. B., J. M. Allsop, H. Granser, A. J. Millett, and I. W. Somerton, 1990, Magnetic interpretation in three dimensions using Euler deconvolution: Geophysics, 55, 8091, doi: 10.1190/1.1442774.

Reid, A. B., D. Fitzgerald, and G. Flanagan, 2005, Hybrid Euler magnetic basement depth estimation: Bishop 3D tests: 75th Annual International Meeting, SEG, Expanded Abstracts, 671-673.

Salem, A., S. Williams, J. D. Fairhead, D. Ravat, and R. Smith, 2007, Tilt-depth method: A simple depth estimation method using first-order magnetic derivatives: The Leading Edge, 26, 1502-1505, doi: 10.1190/1.2821934.

Talwani, M., 1965, Computation with the help of a digital computer of magnetic anomalies caused by bodies of arbitrary shape: Geophysics, 30, 797-817, doi: 10 $.1190 / 1.1439654$.

Talwani, M., and J. R. Heirtzler, 1964, Computation of magnetic anomalies caused by two-dimensional structures of arbitrary shape: Computers in the mineral industries, G. A. Parks, ed., School of Earth Sciences: Stanford University, 464-480.

Thurston, J. B., and R. S. Smith, 1997, Automatic conversion of magnetic data to depth, dip, susceptibility contrast using the SPI ${ }^{\mathrm{TM}}$ method: Geophysics, 62, 807-813, doi: 10.1190/1.1444190.

Williams, S. E., 2005, Comparison of grid Euler deconvolution with and without $2 \mathrm{D}$ constraints using a realistic 3D magnetic basement model: Geophysics, 70, no. 3, L13-L21, doi: 10.1190/1.1925745.

Williams, S. E., J. D. Fairhead, and G. Flanagan, 2002, Realistic models of basement topography for depth to magnetic basement testing: 72nd Annual International Meeting, SEG, Expanded Abstracts, 814-817.

Biographies and photographs of authors are not available. 